Marquette University

e-Publications@Marquette

Psychology Faculty Research and Publications

Psychology Department

$11-1-2010$

Reduction of the Misinformation Effect by Arousal Induced After Learning

Shaun English

Marquette University

Kristy A. Nielson

Marquette University, kristy.nielson@marquette.edu

Post-Print.

Cognition, Volume 117, No. 2 (November 2010), DOI: 10.1016/j.cognition.2010.08.014. 


\title{
Reduction of the Misinformation Effect by Arousal Induced After Learning
}

\author{
Authors: Shaun M. English ${ }^{a}$ \& Kristy A. Nielson ${ }^{\text {a,b.c.* }}$
}

\begin{abstract}
Misinformation introduced after events have already occurred causes errors in later retrieval. Based on literature showing that arousal induced after learning enhances delayed retrieval, we investigated whether post-learning arousal can reduce the misinformation effect. 251 participants viewed four short film clips, each followed by a retention test, which for some participants included misinformation. Afterward, participants viewed another film clip that was either arousing or neutral. One week later, the arousal group recognized significantly more veridical details and endorsed significantly fewer misinformation items than the neutral group. The findings suggest that arousal induced after learning reduced source confusion, allowing participants to better retrieve accurate details and to better reject misinformation.
\end{abstract}

\section{Introduction}

Eyewitness memory has now been empirically studied for more than 30 years. A primary focus of these studies has been on measuring the susceptibility of individuals to the influence of misleading information introduced after the original event (Loftus, 2005). The tendency of individuals to retrieve false information that was introduced after the fact, as if it had actually occurred, is known as the "misinformation effect" (Loftus, 2005). To date, the source monitoring errors hypothesis (Lindsay \& Johnson, 1989) has received much empirical support as the cause of the misinformation effect (Loftus, 2005). It proposes that the sources of witnessed and misleading information become confused, leading to the acceptance of misinformation as accurate because it is misattributed to the witnessed event.

The misinformation effect has been manipulated in a variety of ways. Hypnosis has been shown to exacerbate the misinformation effect (Scoboria, Mazzoni, Kirsch, \& Milling, 2002), and various individual differences can increase susceptibility to it (cf. Loftus, 2005). In contrast, the misinformation effect can be mildly reduced by factors such as when response speed is self-paced vs. speeded (Dodson \& Hege, 2005), the learning context can be reinstated (Thomas \& Sommers, 2005), working memory capacity is large (Watson, Bunting, Poole, \& Conway, 2005), verbal contact with the interviewers is limited (Boon \& Baxter, 2004), multi-modality study is used (Dodson \& Schacter, 2001), and when the delay between learning and test is short, specific types of tests are used, or warnings or feedback is given (cf. Loftus, 2005). Alternatively, 1 English \& Nielson 
a growing memory consolidation literature demonstrates that manipulations introduced after learning can alter later retrieval for such events. To the best of our knowledge, the present study is the first test of the effect of arousal on of the misinformation effect.

Newly acquired memories are highly malleable, and subject to the influence of external factors that may enhance or impair long-term retention (McGaugh, 2000; Meeter \& Murre, 2004; Nielson \& Powless, 2007). This malleability is a consequence of the time required for long-term memory consolidation to occur. Specifically, newly formed episodic memory traces in the neocortex are initially dependent on links within the medial temporal lobe, gradually becoming independent as interconnections between neocortical traces are strengthened (Dudai, 2004; McGaugh, 2000). Consolidation is a complex set of processes that unfold over time after learning, ranging from perhaps minutes (McGaugh, 2000; Nielson \& Powless, 2007) to several hours or days (Revelle \& Loftus, 1992; Walker, 1958), likely varying with task types and demands.

Based on the memory consolidation literature in animals, recent human studies have shown that physiologically arousing treatments administered soon after learning can modulate long-term memory. Specifically, moderate arousal via muscle tension (Nielson \& Jensen, 1994; Nielson, Radtke, \& Jensen, 1996), cold pressor stress (Cahill, Gorski, \& Le, 2003), amphetamine administration (Soetens, Casaer, D'Hooge, \& Hueting, 1995), and negative and positive emotional stimuli (Nielson \& Bryant, 2005; Nielson \& Powless, 2007; Nielson, Vee, \& Erickson, 2005) result in enhanced delayed retrieval of episodic memory in a time-dependent manner. For example, arousal induced via watching a film clip shortly after learning (e.g., oral surgery or comedy) enhanced delayed memory for words that were semantically unrelated to the arousing film (Nielson \& Powless, 2007; Nielson et al., 2005). Furthermore, the surgical and comedic stimuli were equally effective to enhance delayed word retrieval when viewed within 30 min after learning, but a 45-min delay was ineffective (Nielson \& Powless, 2007). Importantly, the effects of arousal are not immediately apparent; perhaps 20 minutes to multiple days may be necessary to measure the modulating effects of arousal on memory retention (Kleinsmith \& Kaplan, 1963; Nielson \& Jensen, 1994; Nielson et al., 2005; Quevedo et al., 2003; Revelle \& Loftus, 1992). The mechanisms of action of arousal effects on memory consolidation may be multi-faceted, but the primary underlying action appears to be modulation of the hippocampal memory system by the amygdala, which is activated by various emotional and arousing treatments (McGaugh, 2004).

While a growing literature exists examining the effect of arousal on episodic memory, few studies have investigated the impact of arousal on source monitoring or false memory. Using arousal manipulated during encoding by incorporating memoranda that were inherently arousing 2 English \& Nielson 
or arousing treatments such as exercise, a few studies have shown that moderate emotional arousal enhances source monitoring accuracy (Doerksen \& Shimamura, 2001; Dutton \& Carroll, 2001; Kensinger \& Schacter, 2006). However, only one study has examined the effect of arousal induced after learning on source monitoring accuracy. It demonstrated that participants who completed a stressful mental task after learning had significantly enhanced later source monitoring accuracy (Smeets et al., 2006). To our knowledge, the effect of arousal on source monitoring or source memory has not yet been specifically evaluated in the context of false memory or a misinformation paradigm.

The present study was designed to evaluate the effects of arousal induced after learning on veridical retrieval and the acceptance of misinformation in an eyewitness memory task. A 2 (misinformation/control) $\times 2$ (arousal/ neutral) between-subjects design was employed where participants viewed multiple film clips that were either followed by forced-choice recognition questions that contained multiple items of misinformation or no misinformation at all (Cann \& Katz, 2005; Tomes \& Katz, 1997). Afterward, participants either watched a negatively arousing or neutral film clip. Retention was measured one week later to allow for memory consolidation to occur. Arousal induced after learning was expected to enhance veridical retention performance and reduce misinformation endorsement (i.e., false memory).

\section{Methods}

\subsection{Participants}

Undergraduate participants ( $n=251,165$ female; mean age $=19.27, \mathrm{SD}=1.92$ ) who received psychology course credit were quasi-randomly assigned to conditions; comparable numbers of participants were assigned to the misinformation $(n=131)$ and control $(n=120)$ conditions and concurrently to the arousal $(n=124)$ and neutral $(n=127)$ conditions, resulting in 59-66 participants per cell. All procedures were approved by the Institutional Review Board.

\subsection{Materials and procedures}

Participants were informed that they would be participating in a study concerning memory for movies. No warning about impending misinformation was given. Prior to presenting film clips, subjective mood and arousal (SMA) was assessed on separate rating scales ranging from 1 (Extremely Negative/Not Aroused at All) to 10 (Extremely Positive/Aroused) (Nielson \& Powless, 2007). Participants then viewed the first of four different 90-s film clips used in previous studies that depicted action scenes ("Z", d'Argila et al., 1969; "Jack's Back” Elwes et al., 1988; "Talons of the Eagle", Hildebrand et al., 1992; "The Big Sweat”, Watkins \& Lommel, 1991; cf. Cann \& Katz,

\section{English \& Nielson}


2005; Tomes \& Katz, 1997; Arentsen, Bock, \& Nielson, unpublished). Immediately following the clip, a 15-item, forced-choice (yes/no) questionnaire was administered to test retention. In the misinformation condition, four of the questions for each film were modified to contain misinformation (Tomes \& Katz, 1997). For each questionnaire item, participants also rated their confidence in their answers on a scale of 1 (Not Confident) to 5 (Very Confident). A 5-min set of surveys (not analyzed) was then administered to preclude differences in rumination. This general procedure was repeated for each film (with order counterbalanced). Participants then completed a second SMA measure.

Arousal was induced after the task using a 3-min video clip of live-action oral surgery, while the neutral group viewed 3-min of a PBS documentary about the link between heart disease and depression (Nielson \& Powless, 2007; Nielson et al., 2005). These clips did not depict actions or content similar to that in the earlier films. A third SMA measure was then administered. After a one-week delay, participants returned and completed an unexpected 15-item yes/no recognition test for each film, in original film presentation order. These assessed memory accuracy (for details unique from the earlier retention test) and misinformation endorsement.

\section{Results}

\subsection{Accuracy of consistent information retrieval}

Group demographic and recognition accuracy data are presented in Table 1. The participants were comparable in age and grade-point average. Immediate memory was less accurate in the misinformation group than in the control group $\left(F(1,246)=22.32, p<.001, n_{p}^{2}\right.$ $=.08)$. The arousal participants, who had not yet been aroused, did not differ from the neutral participants $\left(F(1,246)=1.07, p=.30, n_{p}^{2}=.004\right.$; interaction: $\left.F(1,246)=.42, p=.51, n_{p}^{2}=.002\right)$. Recognition for the films after one week did not differ by misinformation condition for Consistent questions not involving misinformation $\left(F(1,247)=.04, p=.85, n_{p}^{2}=.0001\right)$, but the aroused participants performed significantly better than those who saw the neutral stimulus $(F(1,247)=$ 4.33, $p<.04, n_{p}^{2}=.02$; interaction: $\left.F(1,247)=.27, p=.60, n_{p}^{2}=.001\right)$.

\subsection{Manipulation checks}

Subjective mood significantly changed across the three measurements $(F(2,490)=$ $\left.106.24, p<.001 ; n_{p}^{2}=.30\right)$ and interacted with arousal group $\left(F(2,490)=11.45, p<.001 ; n_{p}^{2}\right.$ $=.05$ ), such that the arousal video caused a decrease in mood compared with the neutral video (Fig. 1A). Subjective arousal also significantly changed across measures $(F(2,492)=11.62, p$ 4 English \& Nielson 
$\left.<.001, n_{p}^{2}=.05\right)$, and interacted with arousal group $\left(F(2,492)=28.34, p<.001, n_{p}^{2}=.10\right)$, showing a significant increase associated with the arousal clip vs. the neutral clip (Fig. 1B). No other effects were significant ( $p s>.20$ ). Arousal change (pre- to post-video) was also correlated with recognition accuracy (Consistent questions) across the sample, $r=.165, p=.009$, while mood change was not $(r=-.006, p=.93)$.

\subsection{Endorsement of misinformation}

The rate of misinformation endorsement of all 16 items was higher in the misinformation group vs. the consistent group, as expected $\left(F(1,247)=59.72, p<.001, n_{p}^{2}=.20\right)$. There was also a lower rate of endorsement by the aroused participants as compared with those who saw the neutral stimulus $\left(F(1,247)=8.19, p<.01, n_{p}^{2}=.03\right)$.

The interaction was also significant $\left(F(1,247)=4.44, p<.05, n_{p}^{2}=.02\right)$, showing a significant difference between the misinformation groups with arousal participants endorsing less misinformation than neutral participants $(p<.01$; Fig. 2). Interaction results were confirmed with Bonferroni-corrected tests: Misinformation/Neutral $(\mathrm{MN})>$ Misinformation/Arousal (MA) $>$ Consistent/Neutral $(\mathrm{CN})=$ Consistent/Arousal $(\mathrm{CA})$, all $p s<.01$ except $\mathrm{CN}$ vs. CA, $p>.10 .{ }^{1}$ The inclusion of sex to the analysis failed to produce any significant main effects or interactions of sex (ps from .24 to .74), although power was too limited to draw strong conclusions.

Because the experimental manipulation caused subjective change in mood and arousal, change scores (pre- to post-video) were regressed to determine whether either or both could predict total misinformation endorsement. The model was not significant in the Control condition, $R=.12, F(2,116)=0.79, p=.45$. However, it was significant in the Misinformation condition, $R$ $=.23, R^{2}=.054, F(2,128)=3.63, p=.03$, where mood change was not a predictor $(p=.27)$, but greater arousal change predicted less endorsement $\left(\beta_{\text {std }}=-.19, p=.035\right)$. Finally, we also identified individuals as "susceptible" to misinformation if they endorsed at least one item in each of the film clips (of 16 items), as has been done in previous studies (Cann \& Katz, 2005; Tomes \& Katz, 1997, 2000). In the Control condition, where no misinformation was given, 38.3\% exhibited this pattern (45.9\% neutral, $30.1 \%$ arousal, $p=.08)$. This differed significantly from the misinformation condition, where $64 \%$ did so $\left(X^{2}(1)=16.68, p<.001\right)$, and where neutral participants exhibited a higher rate of susceptibility $(72 \%)$ than arousal participants $\left(55 \% ; X^{2}(1)\right.$ $=4.28, p=.039){ }^{2}$ Regression in the misinformation condition demonstrated a significant model $(R=.223, F(2,128)=3.3, p=.039)$ where mood change was not predictive $(p=.21)$, but greater arousal change trended toward predicting reduced susceptibility to misinformation $\left(\beta_{\text {std }}=-.17, p\right.$ $=.06)$.

5 English \& Nielson 


\subsection{Confidence}

Confidence was analyzed for consistent and misinformation questions and correct and incorrect answers. Three main effects were significant (other $p s>.05$ ). The arousal group was more confident than the neutral group for correctly answered consistent questions (hits; $F(1,247)$ $=6.2, p=.013, n_{p}^{2}=.03$ ), and correctly answered misinformation questions (correct rejections; $\left.F(1,247)=4.52, p<.05, n_{p}^{2}=.02\right)$. Confidence was also higher in the misinformation group vs. the control group for erroneously answered misinformation questions (endorsements; $F(1,247)=$ $\left.10.41, p<.01, n_{p}^{2}=.04\right)$.

\section{Discussion}

The present study constituted the first test of the effect of arousal on of the misinformation effect. Experiencing a brief arousal stimulus after witnessing events and exposure to misinformation led to enhanced 1-week delayed veridical retrieval of eyewitness memory and to reduced acceptance of misinformation. These findings support earlier studies showing that arousal induced after learning enhances delayed episodic memory (McGaugh, 2000; Nielson \& Bryant, 2005; Nielson \& Powless, 2007; Nielson et al., 2005), and extends them to show a reduction of false memory in an eyewitness task. Importantly, the current study demonstrated that greater subjective arousal change from baseline predicted the degree of misinformation endorsement and trended toward predicting less susceptibility to "habitual" misinformation endorsement.

The present findings also support the literature on the misinformation effect with multiple event paradigms. The rate of misinformation endorsement was consistent with and even slightly higher than in previous studies (Cann \& Katz, 2005; Tomes \& Katz, 1997), possibly because of the additional events and the longer interval between encoding and testing (Higham, 1998). Indeed, one study reported significantly greater misinformation endorsement using a 1-week retention interval compared to shorter intervals (Frost, Ingraham, \& Wilson, 2002).

Although the present study did not directly examine source monitoring, the results are generally consistent with a source monitoring interpretation of the misinformation effect (Lindsay \& Johnson, 1989), and further suggest that arousal might enhance both episodic and source memory. Specifically, the arousal group had greater accuracy and reduced misinformation endorsement than the neutral group, as well as increased confidence specifically for correctly retrieved information and correctly rejected misinformation. Thus, arousal appeared to allow for reduced source confusion and better clarification of actual from suggested details. This 6 English \& Nielson 
interpretation is consistent with previous results showing that arousal after learning is effective to enhance delayed episodic retrieval. Animal and neuroimaging evidence indicates that this enhancement occurs due to direct modulatory effects of the amygdala on memory consolidation via its influence on the hippocampal memory circuit (McGaugh, 2004). In the current study, arousal was induced after the events and misinformation had occurred, supporting that arousal helped keep memory for the video events and later questionnaire information distinct, most likely by enhancing consolidation for both episodic and source information.

While our results support previous findings that confidence is typically a poor indicator of accuracy when misinformation has been presented (Tomes \& Katz, 2000), we also found greater confidence for accurately retrieved information and accurately rejected misinformation in the arousal group. Importantly, one prior study demonstrated enhanced source monitoring accuracy after arousal induction (Smeets et al., 2006). Consistent with this idea, several previous studies showed an approximate average of $10 \%$ improvement in delayed retention due to arousal induced after learning (Nielson \& Bryant, 2005; Nielson \& Powless, 2007; Nielson et al., 2005). The current study demonstrated a comparable benefit in the reduction of misinformation endorsement (see Fig. 2). Thus, the confidence and misinformation results further support the interpretation that arousal enhanced memory consolidation, possibly via modulation of both episodic and source memory. Ideally, future studies would examine the specific effects of arousal on source monitoring, original events, misinformation items, and the respective sources of each item (Cann \& Katz, 2005).

Kensinger (2007) suggested that emotion or arousal specifically enhances memory for source details that have "affective relevance" (p. 215) to emotional stimuli. Yet, studies typically either manipulate arousal during encoding or utilize arousing memoranda. A strength of the current design was that targeted the effect of arousal on memory consolidation, eliminating effects attributable to attention or encoding. Our findings of enhanced veridical recollections and reduced false memory despite any lack of "affective relevance" is consistent with recent episodic memory studies (Nielson \& Bryant, 2005; Nielson \& Powless, 2007; Nielson et al., 2005). Additionally, some studies have suggested that arousal alters central rather than peripheral details of remembered events (e.g., Christianson, 1992). In eyewitness studies, misinformation items often involve peripheral details, which can be of great importance in witness testimony. As Table 2 shows, the misinformation items herein spanned both peripheral and central details of the film events.

Negative stimuli have been suggested to lead to more detailed episodic recall, possibly

\section{English \& Nielson}


due to a "systematic cognitive processing style" that affords greater attention to them (Kensinger, 2007). We employed a decidedly negatively valenced stimulus, but it was presented after encoding and misinformation, when attention could not be influenced. Although it is possible that the negative stimulus was more effective than a positive stimulus would have been, recent studies failed to find valence differences in memory modulation (Nielson \& Powless, 2007) or false memory (Corson \& Verrier, 2007).

In conclusion, the present study uniquely demonstrated that arousal induced shortly after learning allowed better long-term retrieval of episodic details and also allowed better rejection of misinformation. The findings importantly extend the literature on memory modulation to false memory and the misinformation effect, introducing possible implications for developing methods to improve resistance to misinformation.

\section{Acknowledgments}

- The authors gratefully acknowledge the assistance of Timothy J. Arentsen, Theresa Niles, Julie Riederer, Michele Schoenleber, and Marquette University (for financial support).

\section{Notes}

- ${ }^{a}$ Department of Psychology and the Integrative Neurosdence Research Center, Marquette University, Milwaukee, WI 53201-1881, United States

- ${ }^{\mathrm{b}}$ Imaging Research Center, Medical College of Wisconsin, Milwaukee, WI 53226, United States

- ${ }^{\mathrm{C}}$ Department of Neurology, Medical College of Wisconsin, Milwaukee, WI 53226, United States

- $\quad{ }^{*}$ Corresponding author at: Department of Psychology, Marquette University, Milwaukee, WI 53201-1881, United States. Tel.: +1 414288 1796; fax: +1 4142885333.

- E-mail address: kristy.nielson@marquette.edu (K.A. Nielson).

- ${ }^{1}$ Only 10 of the 16 misinformation items "effectively" produced the misinformation effect (i.e., items endorsed greater than chance $p<.05$ criterion; see Table 2). Analysis of these items was nearly identical to the full analysis (Misinformation, $F(1,247)=104.70 . p<.001 . n_{p}^{2}$ $=.30$; Arousal. $F(1,247)=5.30, p<.025, n_{p}^{2}=.021$; Interaction, $F(1,247)=7.73, p<.01, n_{p}^{2}$ $=.03 ; \mathrm{MN}>\mathrm{MA}>\mathrm{CA}=\mathrm{CN})$.

- $\quad{ }^{2}$ Previous studies have only used "effective" misinformation items for this purpose. Although

8 English \& Nielson 
the percentages of "susceptible" individuals differ in using only the "effective" items (16.7\% Control (16.4\% Neutral, 16.9\% Arousal), 36.6\% Misinformation (45.5\% Neutral, 27.7\% Arousal)), all analysis results were comparable.

\section{References}

Boon, J. C. W., \& Baxter, J. S. (2004). Minimizing extraneous, interviewer-based interrogative suggestibility. Legal and Criminological Psychology, 9(2), 229-238.

Cahill, L., Gorski, L., \& Le, K. (2003). Enhanced human memory consolidation with post-learning stress: Interaction with the degree of arousal at encoding. Learning and Memory, 10(4), 270-274.

Christianson, S. A. (Ed.). (1992). Handbook of emotion and memory: Current research and theory. Hillsdale, NJ: Erlbaum.

Cann, D. R., \& Katz, A. N. (2005). Habitual acceptance of misinformation: Examination of individual differences and source attributions. Memory and Cognition, 33(3), 405-417.

Corson, Y., \& Verrier, N. (2007). Emotions and false memories: Valence or arousal? Psychological Science, 18(3), 208-211.

d'Argila, P., Perrin, J., Rachedi, A., Schlumberger, E. (Producers), \& Costa-Gavras, C (Director). (1969). Z [Motion Picture]. France: Cinema V.

Dodson, C. S., \& Hege, A. C. G. (2005). Speeded retrieval abolishes the false-memory suppression effect: Evidence for the distinctiveness heuristic. Psychonomic Bulletin and Review, 12(4), 726-731.

Dodson, C. S., \& Schacter, D. L. (2001). Memory distortion. In B. Rapp (Ed.), The handbook of cognitive neuropsychology: What deficits reveal about the human mind (pp. 445-463). New York, NY: Psychology Press.

Doerksen, S., \& Shimamura, A. P. (2001). Source memory enhancement for emotional words. Emotion, 1(1), 5-11.

Dudai, Y. (2004). The neurobiology of consolidations, or, how stable is the engram. Annual Review of Psychology, 55, 51-86.

Dutton, A., \& Carroll, M. (2001). Eyewitness testimony: Effects of source of arousal on memory, source-monitoring, and metamemory judgments. Australian Journal of Psychology, 53(2), 83-91.

Elwes, C., Moore, T., Blay, A., Kastner, E. (Producers), \& Herrington, R. (Director). (1988). Jack's Back [Motion Picture]. United States: Paramount Pictures.

9 English \& Nielson 
Frost, P., Ingraham, M., \& Wilson, B. (2002). Why misinformation is more likely to be recognised over time: A source monitoring account. Memory, 10(3), 179-185.

Higham, P. A. (1998). Believing details known to have been suggested. British Journal of Psychology, 89(2), 265-283.

Hildebrand, D., Merhi, J., Petersen, C., Ward, K. (Producers), \& Kennedy, M. (Director). (1992). Talons of the Eagle [Motion Picture]. Canada: Shapiro/Glickenhaus Entertainment.

Kensinger, E. A. (2007). Negative emotion enhances memory accuracy: Behavioral and neuroimaging evidence. Current Directions in Psychological Science, 16(4), 213-218.

Kensinger, E. A., \& Schacter, D. L. (2006). When the Red Sox shocked the Yankees: Comparing negative and positive memories. Psychonomic Bulletin and Review, 13(5), 757-763.

Kleinsmith, L. J., \& Kaplan, S. (1963). Paired-associate learning as a function of arousal and interpolated interval. Journal of Experimental Psychology, 65(2), 190-193.

Lindsay, D. S., \& Johnson, M. K. (1989). The eyewitness suggestibility effect and memory for source. Memory and Cognition, 17(3), 349-358.

Loftus, E. F. (2005). Planting misinformation in the human mind: A 30-year investigation of the malleability of memory. Learning and Memory, 12(4), 361-366.

McGaugh, J. L. (2000). Memory - A century of consolidation. Science, 287, 248-251.

McGaugh, J. L. (2004). The amygdala modulates the consolidation of memories of emotionally arousing experiences. Annual Review of Neuroscience, 27, 1-28.

Meeter, M., \& Murre, J. M. J. (2004). Consolidation of long-term memory: Evidence and alternatives. Psychological Bulletin, 130(6), 843-857.

Nielson, K. A., \& Bryant, T. (2005). The effects of non-contingent extrinsic and intrinsic rewards on memory consolidation. Neurobiology of Learning and Memory, 84, 42-48.

Nielson, K. A., \& Jensen, R. A. (1994). Beta-adrenergic receptor antagonist antihypertensive medications impair arousal-induced modulation of working memory in elderly humans. Behavioral and Neural Biology, 62, 190-200.

Nielson, K. A., \& Powless, M. (2007). Positive and negative sources of emotional arousal enhance long-term word-list retention when induced as long as thirty minutes after learning. Neurobiology of Learning and Memory, 88, 40-47.

Nielson, K. A., Radtke, R. C., \& Jensen, R. A. (1996). Arousal-induced modulation of memory storage processes in humans. Neurobiology of Learning and Memory, 66, 133-142.

Nielson, K. A., Yee, D., \& Erickson, K. I. (2005). Memory enhancement by a semantically unrelated emotional arousal source induced after learning. Neurobiology of Learning and

10 English \& Nielson 
Memory, 84, 49-56.

Quevedo, J., Sant' Anna, M. K., Madruga, M., Lovato, I., de-Paris, F., Kapczinski, F., et al. (2003). Differential effects of emotional arousal in short- and long-term memory in healthy adults. Neurobiology of Learning and Memory, 79(2), 132-135.

Revelle, W., \& Loftus, D. A. (1992). The implications of arousal effects for the study of affect and memory. In S. Christianson (Ed.), The handbook of emotion and memory (pp. 113-150). Hillsdale: Lawrence Erlbaum Assoc.

Scoboria, A., Mazzoni, G., Kirsch, I., \& Milling, L. S. (2002). Immediate and persisting effects of misleading questions and hypnosis on memory reports. Journal of Experimental Psychology: Applied, 8(1), 26-32.

Smeets, T., Jelicic, M., Merckelbach, H., Peters, M., Fett, A., Taverniers, J., et al. (2006). Enhanced memory performance on an internal-internal source monitoring test following acute psychosocial stress. Behavioral Neuroscience, 120(6), 1204-1210.

Soetens, E., Casaer, S., D'Hooge, R., \& Hueting, J. E. (1995). Effect of amphetamine on long-term retention of verbal material. Psychopharmacology, 119(2), 155-162.

Thomas, A K., \& Sommers, M. S. (2005). Attention to item-specific processing eliminates age effects in false memories. Journal of Memory and Language, 52(1), 71-86.

Tomes, J. L., \&Katz, A. N. (1997). Habitual susceptibility to misinformation and individual differences in eyewitness memory. Applied Cognitive Psychology, 11(3), 233-251.

Tomes, J. L., \& Katz, A. N. (2000). Confidence-accuracy relations for real and suggested events. Memory, 8(5), 273-283.

Walker, E. L. (1958). Action decrement and its relation to learning. Psychological Review, 65, 129-142.

Watkins, J. (Producer), \& Lommel, U. (Director). (1991). The Big Sweat [Motion picture]. United States: AIP Home Video.

Watson, J. M., Bunting, M. F., Poole, B. J., \& Conway, A. R. A. (2005). Individual differences in susceptibility to false memory in the Deese-Roediger-McDermott paradigm. Journal of Experimental Psychology: Learning, Memory, and Cognition, 31(1), 76-85.

11 English \& Nielson 


\section{Appendix}

Table 1

Group demographics and accuracy (mean ( \pm SO)).

\begin{tabular}{lllll} 
Group & Age (years) & GPA (of 4.0) & \% Correct consistent items $\sim($ 1st session) & \% Correct consistent items $\sim($ delayed) \\
\hline C/N $(\mathrm{N}=61)$ & $19.9(2.8)$ & $3.2(0.5)$ & $84.3(5.5)$ & $75.1(7.5)$ \\
C/A $(\mathrm{N}=59)$ & $19.2(1.3)$ & $3.2(0.5)$ & $84.5(4.8)$ & $77.3(5.8)$ \\
M/N $(\mathrm{N}=66)$ & $19.0(1.8)$ & $3.4(0.4)$ & $80.7(5.7)$ & $75.7(6.0)$ \\
M/A $(\mathrm{N}=65)$ & $19.0(1.9)$ & $3.3(0.5)$ & $81.8(4.8)$ & $77.1(7.7)$ \\
& All ns & All ns & M: $p<.01^{\mathrm{a}}$ & A: $p<.05^{\mathrm{b}}$ \\
& & & Others ns & Others ns
\end{tabular}

Conditions: $\mathrm{C}=$ control, $\mathrm{M}=$ misinformation, $\mathrm{A}=$ arousal, $\mathrm{N}=$ neutral; $\mathrm{GPA}=$ self-reported grade-point average; Consistent items contained no misinformation, thus they evaluated only veridical retrieval from the films.

${ }^{\mathrm{a}} F(1,246)=22.32, n_{p}^{2}=.08$.

${ }^{\mathrm{b}} F(1,247)=4.33, n_{p}^{2}=.02$.

Table 2

Misinformation endorsement compared between groups (mean ( \pm SEM)).

\begin{tabular}{|c|c|c|c|c|c|}
\hline Item & Description & Misinfo & Control & $t(249)$ & $\mathrm{p}$ \\
\hline BS 1 & One of the robbers has long hair & $0.45(0.04)$ & $0.32(0.04)$ & -2.2 & .03 \\
\hline BS 3 & Robbers took two black suitcases & $0.38(0.04)$ & $0.37(0.04)$ & -0.3 & .81 \\
\hline BS 5 & Robber had sunglasses & $0.36(0.04)$ & $0.48(0.05)$ & 1.9 & .054 \\
\hline BS 9 & Presence of male bank teller & $0.23(0.04)$ & $0.12(0.03)$ & -2.4 & .02 \\
\hline Z 3 & Victim charged by white vehicle & $0.53(0.04)$ & $0.43(0.05)$ & -1.6 & .11 \\
\hline Z5 & Three "thugs" approach the victim & $0.63(0.04)$ & $0.43(0.05)$ & -3.2 & $<.01$ \\
\hline Z 7 & Attacking "thug" holding a stick & $0.30(0.04)$ & $0.27(0.04)$ & -0.6 & .56 \\
\hline $\mathrm{Z} 10$ & 1 victim companion had clipboard & $0.66(0.04)$ & $0.28(0.04)$ & -6.7 & $<.001$ \\
\hline ToE 2 & Pick up truck was blue & $0.68(0.04)$ & $0.52(0.05)$ & -2.7 & $<.01$ \\
\hline ToE 4 & Airplane was American Airlines & $0.44(0.04)$ & $0.48(0.05)$ & 0.5 & .61 \\
\hline ToE 7 & Victim was carrying a purse & $0.66(0.04)$ & $0.36(0.04)$ & -4.9 & $<.001$ \\
\hline ToE 10 & Woman and child in the terminal & $0.64(0.04)$ & $0.29(0.04)$ & -5.9 & $<.001$ \\
\hline JB 2 & Color of pick up truck (brownish) & $0.47(0.04)$ & $0.28(0.04)$ & 3.1 & $<.01$ \\
\hline JB 6 & Small stool used as a weapon & $0.50(0.04)$ & $0.33(0.04)$ & -2.8 & $<.01$ \\
\hline JB 10 & Guns lie on a white carpet & $0.18(0.03)$ & $0.10(0.03)$ & -1.7 & .09 \\
\hline JB 13 & Unmarked police car; blue lights & $0.40(0.04)$ & $0.19(0.04)$ & -3.8 & $<.001$ \\
\hline
\end{tabular}

Note: $\mathrm{BS}=$ The Big Sweat; $\mathrm{Z}=\mathrm{Z} ; \mathrm{ToE}=$ Talons of the Eagle; JB = Jack's Back; Misinfo = Misinformation . 


\section{Figure 1}
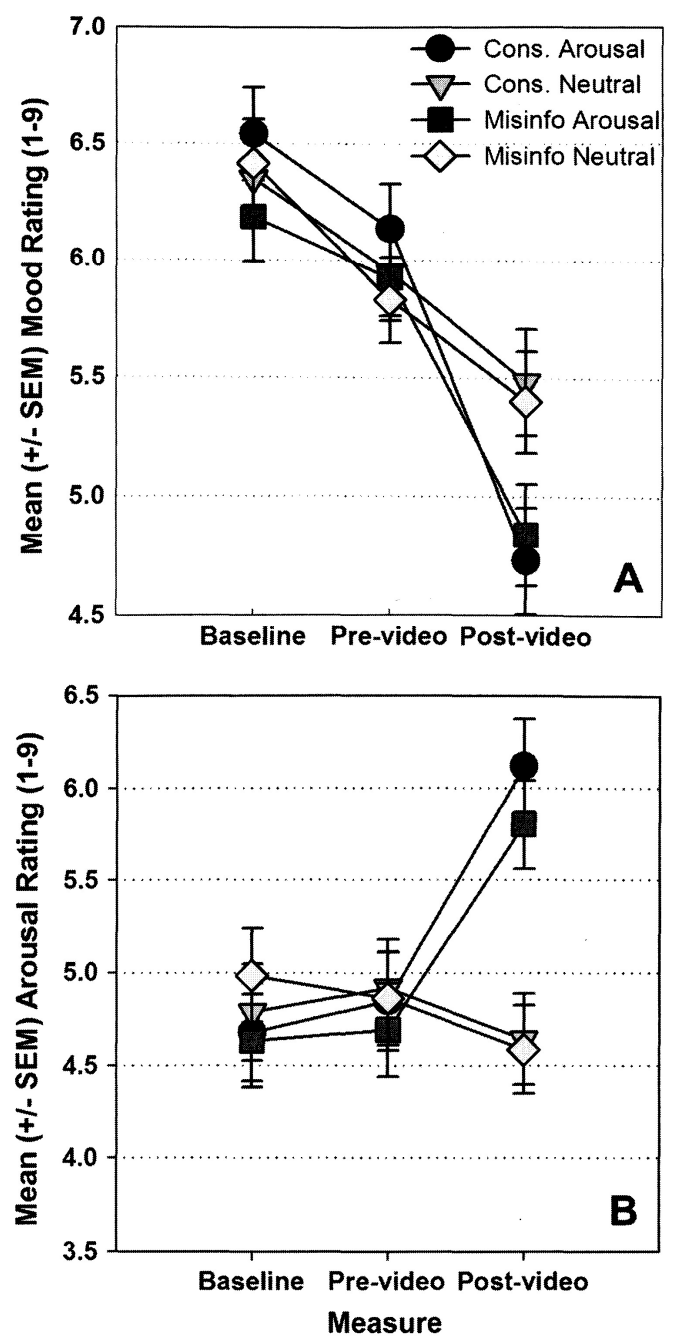

Mean ( \pm SEM) subjective mood ratings $(A)$ and subjective arousal ratings $(B)$ are depicted by each group for baseline, pre-video, and post-video measurements. Arousal participants had significantly decreased mood and increased arousal after the manipulation compared with neutral participants. 


\section{Figure 2}

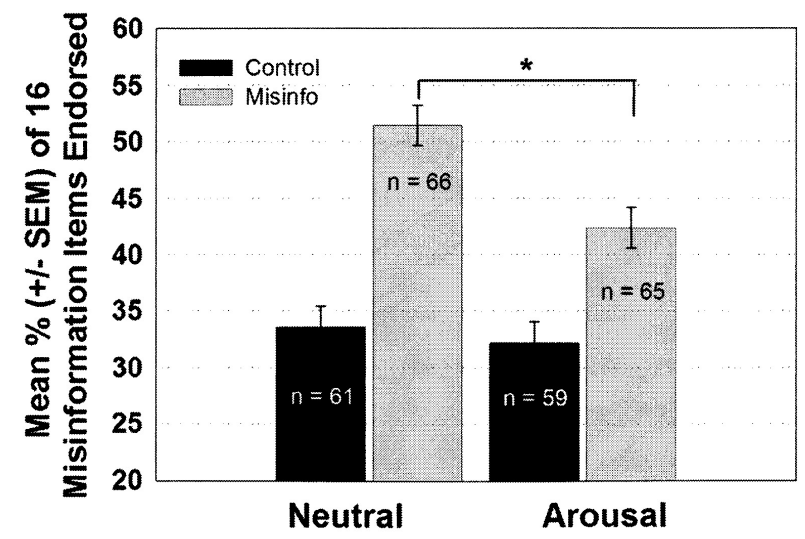

Mean ( \pm SEM) percentage of misinformation items endorsed in the control condition (i.e., where no is misinformation given) and in the misinformation condition, separated by arousal and neutral groups, after the one-week delay for the 16 misinformation items. Arousal after the task significantly reduced misinformation endorsement in the misinformation condition in both analyses. Results were comparable using only the 10 "effective" misinformation items. 